\title{
Propuesta de auditoría de recursos de información en Internet: SCIEL 0 y la ciencia perdida
}

\author{
MARÍA Do Lo RESAYu So GARCíA \\ Departamen todeIn forma cióny Documen tación, Facul taddeComunicacióny \\ Documentación,UniversidaddeMurcia,España. \\ E-mail:mayu@um.es \\ Victoria Martínez NaVARro \\ DepartamentodeIn formaciónyDocumentación, FacultaddeComunicacióny \\ Documentación,Universidadde Murcia,Comunidad Au tónomadela \\ Región de Murcia, Es paña. \\ E-mail:vicmarti@um.es
}



\section{RESUMEN}

El presente trabajo evalúa un recurso de información publicado en Internet es ta ble cien do como re fe ren te el con cep to de au di to ría. El re curso esSCIELO (Scien tific elec troniclibraryon line), un mo deloparala publica ción electróni ca en paí ses de Amé ri ca La ti na y el Ca ri be. La se lec ción se debe al va lor que SCIELOpresen tacomoins trumen to paralograrunaproducción cien tíficanacio nal más visible y ac ce sible por me dios elec tró nicos, y al mis mo tiem po desa rro lla una base de da tos conla cual se pue de evaluarla pro duc ción cien tífica de un país y aumentar su visibilidad internacional.

Se parte del concepto de auditoría, método que incluye la evaluación de recursos di gi ta les. Las con clu sio nes del tra ba jo po nen de ma ni fies to la im portan cia de uno de los es ce na rios de la eva lua ción de re cur sos de in for ma ción: la au di to ría, como me dio para de tec tar los pun tos fuer tes y dé bi les del re curso. En el caso se lec cio na do la aten ción se cen tra en la pro pia eva lua ción de la producción científicadelos países endesarrollo,yespecialmentede América Latina y El Caribe. El proyecto nace para cubrir el vacío en la producción cien tí fi ca de ha bla no in gle sa, y parain ten tar pa liar el fe nó me no de la cien cia que se pierde por falta de medios de difusión científica.

Pa la bras cla ve: Produc ti vi dad cien tífica, La ti noa mérica, Fuen tes de in for ma ciónen In ter net, Eva lua ción de la in for ma ción, Au di to ría, Eva lua ción de re cur sos, In di ca do res de cai dad, Recur sos de in for ma ción, Scielo recur soelectrónico. 


\title{
86 InvestigacónBiblictedoógia v. 18 N o. 37 julio/ diciembre de 2004
}

\section{AUDIT PROPOSAL FOR INFORMATION RESOURCES IN INTERNET: \\ SCIELO AND LOST SCIENCE \\ MARÍA DOLO RESAYUSO-GARCÍA \\ VICTORIA MARTÍNEZ-N AVARRO}

\begin{abstract}
The articleap praises an in forma tion re sourcepublished in Internetusing as ref er ent the con cept of audit. The re source is SCIELO (Scien tific Electronic Library On line) a model for elec tronic pub li ca tion in Latin Ameri can and Carib bean coun tries. SCIELO was cho sen be cause it was thought valuable as an instrument that can help to achieve a more visible and accessible scientific national production by means of electronic media. It also de vel ops a data base with which the sci en tific pro duc tion of a country may be as sessed and whose in ter na tional visibility can be in creased.

The scanning method includes a digital resources assessment. Conclusions manifest the importance of inspecting information resources as a means of high light ing weak and strong points in the se lected case which cen ters its at ten tion in the as sess ment of the sci en tific pro duc tion of developing countries, especially in Latin America and the Caribbean. The projectintends to diminish the phenomenonoflostscientificproduction which is not in English due to the lack of proper scientific diffusion. Key words: Sci en tific produc tiv ity, La ti noamerica; Sources of in for ma tion in Inter net, Information evaluation, Auditing, Quality indicators, Information re sources, SCIELO Electronic re source.
\end{abstract}

\section{INTRODUCCIÓN}

$\mathbf{E}$ l escenario actual de las fuentes de información digitales es la publicación de ellas en Internet. Este he cho no ha afec ta do sola men teal mun do de las fuen tes dein for ma ción sino tam bién al dela co mu ni ca ción y or ga ni za ción del co no cimiento en general y del científicoenparticular,enriqueciéndolos y transformándolos. A tra vés de laWWW sepue de te ner ac ce so a una va ria da tipología dere cursos: bases de datos,publicacionesylibros digitales, in formaciónturística,información comercial, ca tálo gos debiblio te cas, et cé te ra, y ello sin ha cer re feren cia a la am pliagama ti pológica de Literatura Gris, warkingpapers prepint, repart, y disausión papers La oferta de consulta de fuentes y recursos de información en línea crece cada día, al grado de que ya no exis te ins ti tu ción o ser vi cio edi to rial que se pre cie que no dis pon ga en In ternet, ya sea el tex to com ple tode sus servicios obien ofrezcain forma ción re ferencial sobre ellos.

Enunaprimeraaproximación pue deafirmarsequeun recursodigitaldecalidades aquel que com bi na un buen con tenidoy una buena forma o una bue na organiza ción de este con te nido, pero esa duali dad analíti ca ya está su frien do críticas. Sin em bargo evalua mos aquíes tos dos as pec tosesen cia les de todore cursoweb, lo cualim plicaun cono cimien to en pro fun didad so brelas ca rac te rís ticas delas fuen tes y re cursos digitales publicados en Internet, siempre en el contexto de la evaluación comparativa 
(bendhmarking.Dichasca racterís ticas podrían re su mirseen tres:larecuperabilidad,la capacidad,ylahipertextualidad.

Uno delos es ce na rios del tra ba jo dela evalua ción es pre cisa men tela au di to ría.Se evalúa elob je to que se va a au ditar: una fuen te, un re cursodigital oun proyec to deinfor ma ción. Laevalua ción de un re curso de in forma ción permi te detectarlos puntos fuer tes y débiles deéste y proponerme di das correc to ras que mejo ren lacalidad dela fuen te en re la ción con las de su en torno al ofreceral terna tivas. Deesta forma re sul ta habitualquela au ditoría de in formación pre cisela evalua ción deuna fuenteen elconjun to de aquéllas con las que se her ma na, de tal for ma que se in for me cuál es su po sicionamiento en el sistema. La auditoría, concepto amplio y que incluye a la evaluación, tambiénayu daa es tructu rarproyectos de desarrollodepublicaciones digitales, que espre cisamenteloque pretendemosabordareneste ar tículo alauditarelProyec toScielo,peroan tesjustifiquemosla elección deesterecurso.

Es sabido que el resultado de la investigación científica es comunicado y validado principalmente a través de la publicación en revistas científicas y evaluado por pares. Sinembargomu chas revis tas científicas deestasregionesen fren tangraves dificulades de dis tribucióny di fusión, lo que limita el accesoy eluso delain forma ción cien tfí cagenerada,loquea to dasluces esinjus to. Estasitua cióndificul taade más elacce soadecuado y actualizado a la información científico-técnica, con las repercusiones que esto conlleva para el desarrollo económico y social de un país. Pero sobre todo, y esto es muy impor tante, en torpe ce el apoyoalos proce sos de toma de de cisio nes en es tos paí sesen rela ción conla pla nifi ca ción, la formula cióny la aplica ción depolíticas públicas, incluidas aquellas destinadas a fomentar la práctica profesional en cualquier ámbito científicoytécnico;esdecir,enI+D + I(Investigación,DesarrolloeInnovación).

Hemosconstatado que una de las dificultades de es tos países para es ta ble cerestra te gias depolíticas científicas es pre cisamen tela fal ta de ba ses de da tos quepermitan co nocerla producción cien tífica en un con tex to más am plio, la eva lua ción delimpacto de su producción a nivel local e internacional y, lo que es más importante, la percepcióndeladinámicadecirculacióndelainformacióncientífica.

Sädoesunmodeloparalapublicación electrónica cooperativaderevistascien tíficasenInternet, desarrolladoespecialmentepara responderalasnecesidadesdelacomunicacióncientífica en los países de Amé ri ca La ti na y el Ca ribe. Es éste uno de los proyectos sobre bibliotecas virtuales científicas de Latinoamérica que tiene mayor repercusiónenelámbitocientíficosa nitarioespañol.Elproyectoen sísees tudia rá en el pró xi mo epí grafe, de tal for maque sola men te pretende mos en estain tro ducción justificar por qué se ha seleccionado este recurso digital para poner en práctica una auditoría.

La ra zón fun da men tal que ha sus ten ta do la se lec ción de este re curso tie ne su ori gen en los prin ci pios que fun da men tan el pro yec to y que, a nues tro jui cio, son un claroejemplodelos cambiosacontecidos en elproce so delacomunicación científicade es tosúl timos años (Ayu so García y Martínez Na va rro 2003ayb), y con tribu yena de- 


\section{InvestigacoónBiblictecoógia v. 18 N o. 37 julio/ diciembre de 2004}

sarrollarelnuevoparadigmadela documen ta ción cien tíficay téc nicay, sobre todo, a quelacienciagenera daen elámbitonoanglo sajón no seauna "cienciaperdida".

El pro yec to se fun da men ta en el uso de las TIC(Tec nologías dela In forma ción y Comunicación)y enlos proce sos decomunica ción científica, yaprovechalas presta ciones que tiene la publicación digital frente a la analógica a la vez que establece un método estándar de trabajo para publicar colecciones digitales de publicaciones científicasmejorandosuvisibilidadyaccesibilidad, ycontribuyendoamejorar su factordeimpacto.

\section{AUDITORÍA DE FUENTESY RECURSOSDE INFORMACIÓN PUBLICADOS EN INTERNET}

\section{L a auditoría de recursos informativos}

A grossomodpode mos de cirquela au dito riapuedeser de finida de for ma genérica como un instrumento que permite establecer el referente, el modeloyelposiciona mien todelaorganización, ins titu ción, empresa, etcétera, conelobje to medido(evaluado), y analizar el impacto y los resultados de tal medición. Para algunos autores, en tre los que des ta ca mos a Hen riet, Fourgous y Beau-Dau del, la au di to ría tie ne la finalidad de evaluarel recursocomouna to talidad encuan to a su funciona mien toyuti lidad co ti diana. Poruna par te una au dito ría se sitúa enla en cru cija daen tre elpasado, ya que eva lúa la efi ca cia y los re sul ta dos lle va dos a cabo, y por la otra, en las re percusiones so bre el fu turo, al in ten tardi se ñarca mi noseitine ra rios progre sivos; esdecir, al es ta ble ceruna pro pues ta para mejo raro, en su caso, es ta ble cer una pro pues ta dis tinta.Estoes, unconocimientonuevo.

Auditarunre cursoconsisteenanalizary eva luar, con este dobleobje tivo; es decir, detec tarlospuntos fuertesy débiles ypoten ciarlos primerosysubsanarlossegundos para de estemodo me jo rarlages tión dela fuen te o el sis te ma de in forma ción digital. ${ }^{1}$ Elobje tivo úl timo es mejorarla pues taen escena delsis tema; enotras palabras, laaudi to ría de un re curso per si gue op ti mizarsu uso den tro de un con tex to de ac tua ción, sea éste una organiza ción, institu ción, un colec tivode usua rios, o, como su cedeen el caso que nos ocupa, la política de las pu bli ca cio nes cien tíficas de un país, y, acto se guido, ofrecerunapropues tanovedo saquecontribuyaaldesarrollodelas fuentes del conocimiento(AyusoGarcía,2001).

La fun damenta ción de es tas au dito ríasparte delalitera tu racien tífica de es tudiosos so bre el tema como Eli za beth Orna $(1990,1994,1996,1999)$ y más re cien te menteSueHenczel(2001 y2000), porcitaralosmásrepresentativosyactuales.

1 Partedeesta me to dología fuepresenta da porla doc to ra.Dolores Ayu so García comoponen te del Módulo "Au ditoría derecursosin formativos y sistemas dein forma cióndigital.Publicaciones digi talesynormalizaciónderecursos" enla diploma tu ra "Sis temas y Serviciosdein formacióndigital", organizado por la Pontificia Universidad Javeriana de Bogotá.Colombia, Junio 2003. 
Estas premisas, consideradas como básicas, sustentan el análisis y la evaluación posteriordelrecurso,ypodríansintetizarsecomosigue:

* La información es un recurso y como tal ha de ser gestionada. En dicha gestión en tra en jue gola po lítica de in for ma ciónyla au di to ría como es ce na rio de la evaluación de una fuente de información.

* La evalua ción de un re cur so dein for ma ción se vin cula con los obje tivos dela orga ni za ción que os ten ta la au to ría de la fuen te y con el per fil de usua rios a los que se dirige.

* Enrelación conla premisaan terior,puedeavanzarseunatercerayúltima según la cual la auditoría de una fuen te o recursodein forma ción se realiza siem preen relación con los objetivos corporativos. Por tanto la auditoría determinará el valor del recur so den tro de la or ga ni za ción y de fi ni rá su rol fun cio nal y su utilidad en el sistema de información corporativo en el que se incardina.

Según estas premisas la auditoría de una fuente de información podría sintetizarse en el proce so de análi sis yla eva lua ción pos te rior de ésta, con elobje to de mejorarsu uso enun con tex to de termina do, yen aportarmejoras al sis te ma si ello fuera preciso. La definición y concreción de dicho contexto en cada caso particular permitiráajustar el proceso de estudio, la identificacióny la evaluación, con la inten ción deincrementarla con sisten cia y calidaddelafuente,permitirsucompatibilidadyfacilitarsurecuperación.

Ahorabien, resulta de cru cialim portanciano perderdevis ta que nos es ta mos refiriendoala evalua ción de fuen tes y re cursos dein for ma ción digitalesenelcon tex tode la in forma ción cien tíficay téc nica, comomedio para acre ditarla calidad yvalidez de lain forma ciónfacilitadaporlospro fesionalesdeladocumentacióncientífica.Y desdeesteobjetivo, elpresen tetrabajointen taproporcionaruna metodologíadeevaluación que nace del estudio de un amplio conjunto de aspectos relacionados con las fuentes de in for ma ción y la World Wide Web, y que sin em bar go se cen tra es pe cialmenteentrescuestionesesenciales:

* Características y propiedades de la fuente de información digital frente a la analógica.

* Estructura de la fuente o recurso de información digital en línea.

* Novedadesqueaportaparala organización, consul tay difusión delainforma ción que contiene.

Yalametodologíatradicionalde fen didaporBeaudiquez(1998:34-38), orientabala evaluación de fuentes analógicas ha ciala ta reanecesariadevalorarunaseriedeelementos intrínsecos a tra vés de los cua les se exa mi na hoy el tipo de fuen te de in for ma ción ante la que nos en con tramos. Vemos portan to que evaluarno es un con cep to nuevo, pues ya seana li za bala co ber tu ra, la ac tua liza ción, la pre sen ta ción física, el tratamiento quedaalain forma ción, etcétera Noobstante,las caracterís ticas es pecíficas delas fuentes digitales en línea ha cenque suanálisis y su evalua ción presen tenuevos re tos yque se cen tren en los as pec tos for ma les y en la ca li dad de los con te ni dos del re cur so. Se trata 


\section{Investigacón Biblictecoógia v. 18 N o. 37 julio/ diciembre de 2004}

pues de unatarea que re quie re una me to dología nueva, es pe cíficay propia, que guarda cier ta re la ción con lain for ma ción del pro pio re cur so. En ella, el pro fe sio nal de la documentación no solamente identifica y recupera información de Internet, sino que pro por cio na una des crip ción de valor aña di do so bre re cursos digita les que por su naturaleza requieren de un control documental y ofrecen, en realidad, un documentonuevo.

Parece lógico, por tanto, que se desarrollen modelos eficaces para evaluar una fuen te de in forma ción en la Red. Son mu chos los proyec tos lle va dos a cabo con esta finalidad, en trelosquedes ta ca mos la inicia tiva parala normalización delos sis temas de metadatos, Dublín Core Metadata Iniciative, la importancia cada vez mayor de agencias evaluadoras (information gateways) Physical Sciences Information Gateway (Psiga te) ${ }^{2}$ y otras pro pues tas más teó ri cas, en tre las que se en cuen tran por su exhaus tividady amplitud tipológicala de AlisonCooke(2001). ${ }^{3}$ Sin em bargo uno de los que ha con ta do con ma yor acep ta ción ha sido el delISI (Ayu so Gar cía, 2001) que ha de sa rrolla do una me to do lo gía de evalua ción de re cursos web orien ta dos hacia el ámbito académico y de investigación, con el objeto de in cluir los en el Cu rrentWeb Con tents, y elque tam bién he mos ele gi do por la fa ci li dad de su uso y la com pren sión porpar te delos pro fe sio na les y, lle ga do el caso, como una pro pues ta de valor aña didoasu servicio, in clu so de au to evalua ción. AlISI sele hanin corpora doparámetrose in dicado res de calidad que sehan ade cua doa los obje tivos de este trabajo. Ental metodologíasedefinencomocriterios deevaluaciónlossiguientes:

* La au toridaddel re curso web. Estoes, la cre dibilidaddel au toren re ferenciaal contenido de la página, especialmente si estamos ante una página oficial. La objetividad de la página, laiden ti fica ción del au tor yla po si bilidad decon tac tar con él.

* Laprecisión del recurso. Paráme tro que seen cuentraen es tre charelacióncon el anterior pues to quela au to ría de una pá gi na se en cuen tra vin cula da necesa ria men te con la au to ri dad de ésta. De esta for ma un buen nivel de au to ríain di ca una información objetiva y precisa.

* La actualidad del recurso web, o puntualidad en la información facilitada.

* La funcionalidad en el diseño y la navegación del recurso. La presentación estructuradadelain formación, yundiseñogeneraldefácilusoyes tructuralógica.

*a aplicabilidad y contenido del recurso en relación con otros productos.

* El alcance y propósito de la fuente.

2 Psigate: PhysicalSciences In formation Ga teway (fechade con sulta:20 de di ciem bre 2003. Dis ponible en do cu men to web http://www.psiga te.ac.uk)

3 Véa se los tra ba jos de Kirk, E (1997), http://www.mil ton.mse.jhu.edu:8001/re search/edu ca tion /net.html; Gris, Terry A. (1996) http://www.daphne.palomar.edu/TGSEARCH; Tillman, E. (1997), http://www.tiac.net/users/hope/findqual/htm, y otros. 
* Elniveldeaudienciaenlaco munidad científicaoenlainves tiga ción académi ca, ámbitos en los que centra ISI su trabajo de referencia de fuentes de información.

* Lacalidadenlaescritura.Los editoresbuscanortografíabásicaycompetencia gramatical con un uso mínimo del argot. Se valora la claridad en la discusión de cuestiones complejas y/o la genialidad, aunque ésta no es nada común.

* Crí ti cas. Los pre mios en la web se con ce den por va rias ra zo nes, des de el número de visitantes por página hasta la calidad del contenido académico.

A partirde esteproyecto seha desarrollado unprotocolodeevaluacióndefuentes y recursos dein formación publica dos enInternetque sirve comohe rramientadetra ba jo para au di tarlos re cur sos que con tri bui rán a la re dac ción de un do cu men to nuevo yaunnuevoco nocimien to. EstePro to colo, cuyame to do lo gía nace deladenomi nada "arquitectura de la información", ${ }^{4}$ examina los aspectos de organización y es truc tu ra de la fuen te de in forma ción, los as pec tos de en ca je deésta en el con tex to glo baldelaRed,y porúl ti mo suusabilidad. Se tra ta de darun paso cualita tivo yconfigurarunapropuestanueva.

Elproceso de la evalua ción, que se cen tra eneste trabajo en el es ce na rio dela au ditoría de fuen tes de in for ma ción, da co mienzo con la ex plo ra ción de la fuen teconla in ten ción de de terminarlos ob je tivos, los te mas, yel sec tor del públicoalqueva destina da; es decir, des ta ca mos los usua rios rea les y po tenciales. Enesta prime ra fase el ana lis talle va a cabounaiden ti fica ción dela fuen te, a par tirdela vi si ta y el exa men somero dela página principal de tal fuente, y de unos dos niveles dena vegaciónporsección. Con los datos recogidos yaes ta ría en con di cio nes de de ter minarel al can ce temático de la fuente, que será expresadomediantepalabras clave susceptibles de ser rectificadasalfinaldelprocesodeevaluación.

En una segunda fase el analis ta/au ditorproce deya aunaex plo raciónajus tadaal proceso de evaluación que pro po ne el Pro to colo de las au to ras. Aquí por tan to comienza el reconocimientoy cóm pu to de cada uno de los pa ráme tros e in dicadores, que se gún la Figura1 con forman los cues tio na rios de evalua ción (dhekklist) del análisis porsecciones de la fuen te. Finaliza da esta fase de trabajo, el ana lis ta se en cuen traen disposición de esbozar las conclusiones (puntos fuertes y débiles de la fuente de información) y de ha cerlas reco men da ciones que pro po ne para mejo rar el rol funcionalylautilidaddela fuenteen elsis tema dein formacióncorporativo, siempreteniendoencuentaelusuariofinalypotencialalquevadirigido.

4 Ron sen feld,L. y Mor ville,P. (1998): Informationarditectureforthewaddwideweb Cam bridge: O'Reill. 
Figura 1

Diagrama de Auditoría de recursos de información en Internet

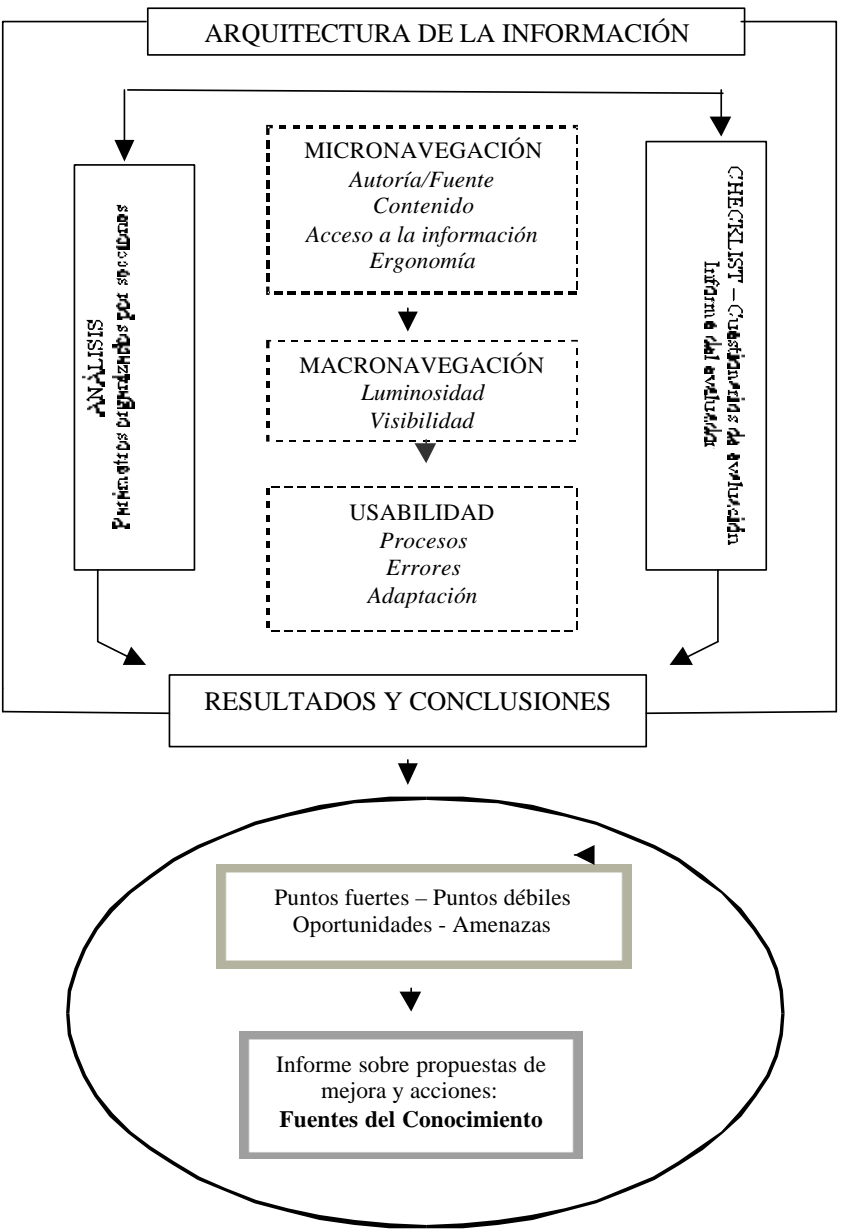

Elaboración propia: Ayu so Garcíay Navarro Martínez

\section{Análisis del proyecto Scielo}

El fenómeno deno mina do "cien cia perdida" es la en fermedad quehaaquejadoa lainves tiga cióncientíficala tinoamericanaduranteaños,ycondenadoalanonimatoa la ma yo ría delos es tu dios de sa rro lla dos en esta región. Elproyec to Scielonace con el obje tivo prio ri ta rio de mejo rarel nivel de di fu sión dela produc ción cien tífica de los países ende sarrollo, particularmen te de AméricaLa tinay ElCaribe, y de ase gurar la visibilidadyelaccesouniversalasuliteraturacientífica. 
Scielo es una biblioteca científica virtual, especializada en publicaciones periódicas, quenació en 1997 abarcan douna colec ción ya selec cio na da derevis tas es pecializadas bra sileñas. Elpro yec to es el re sul ta do dela co o pe ra ción en tre BIREME (Centro La ti no ame ri ca noy del Caribe deIn forma ción en Cien cias de la Salud) ${ }^{5}$ y FAPES (Fundación de Apoyo a la Inves tiga ción delEs ta do deSao Paulo) ${ }^{6}$, con el objetode desarrollarunméto doparala publica ción, al ma cenamien to, recu pera cióny evalua ción de publicaciones digitales con el uso de las TIC en el entorno de la Web Wide Web. El res pon sa ble es pañol es la Bi blio te ca Na cio nalde Cien cias dela Salud, merced a unacuer do de cola bo ra ción en trela OPS(Organiza ción Panamericana delaSa lud) / OMS(OrganizaciónMundialdelaSalud)yelIns titu todeSaludCarlosIII.

Fapespes una fun da ción na cida en 1970 y que per sigue au men tar la vi sibilidad de la produccióncientífica nacional. Por su parte Bireme se fundó en 1967 como la Biblioteca RegionaldeMedicina, a partirdel convenio firmadoentreelGobiernodeBrasil y la OPSen São Paulo. Setra ta deuncen tropertene cien tealaOPS y que tra ba ja como ofi ci na de laOMSparael continen teamericano.FapespyBireme, en colaboracióncon editorescientíficos, desarrollaron una me to do logía co mún para la publica ción, re cuperación, ydifusión delas revis tas digitales, me todologíaquehadadosustento a todo el pro yec to dela Biblio te ca Virtual en Salud: Scielo. A partirde 2002, el Pro yec to tiene el apo yo del CNPq-ConselhoNacionaldeDe senvolvimentoCientíficoe'TecnológicodeBrasil.

El proyec to se ini ció en marzo de 1997, obe de cien do a las de man das y ex pec ta tivas que seiban crean do en el seno dela in ves tiga ción bra sileña, ein ten ta pro moverla vi sibilidadyaccesibilidaddela produccióncien tífica bra sileña, alavez que bus ca paliarlaconstatacióndedos realidades:

* Laescasapresenciadelaliteraturacien tíficabrasileñaenlosíndices yrepertorios in terna cio na les. A este res pec to, y to man do como re feren te el nue vo mi lenio, puede afirmar se que en el año 2000 , sola men te 12 revis tas la ti no americanas y del Caribe con ta ban con pre sen cia en el Jaumal Citation Reparts del ISI (Institute for Scientific Information).

* Lasbibliotecas electrónicasmejoranlaacce sibilidadala produccióncientífica a nivel in ternacionalpor con tarcon pres ta ciones devaloraña dido, inherentes a la na tu ra le za di gi tal de la Red, tan to en la edi ción, como en la con sul ta y re cuperación de la información.

Se gún estola bi blio te ca vir tual Scie lo nace en ton ces con ob je tivos cla ros y pre cisosqueproponen: eldesarrollo y es tan da riza ción de una me todología de edición digi tal de la pro duc ción cien tí fi ca; la pues ta en mar cha de un servi cio web de información

5 Bireme [Fecha de ac ce so 10 sep tiem bre 2003]. Dis po nible en el do cu men to web: http://www.bireme.br.

6 IReuniónRegional delaRedSäloInternationalConference on ScientificElectronic Publishingin Developing Countries. [Fecha de acceso 20 diciembre 2002]. Disponible en el documento web: http://www.icsep.info/. 


\section{InvestigacónBiblictedoógia v. 18 N o. 37 julio/ diciembre de 2004}

especializadaenpublicacionesperiódicas, yaseamediantelapublicaciónelectrónica de re vis tas cien tíficas o bienla orga niza ción deba ses de da tos fac tuales ybibliográficas; y la definición de unos indicadores estadísticos que midan el impacto de dicha literatura.

En una primera fase del proyecto, comprendida entre marzo de 1997 y mayo de 1998, los es fuerzos secen traronenelde sarrollo deuna metodo logía de tra bajoque se puso en prác ti ca con una co lec ción de diez re vis tas bra si le ñas perte ne cien tes a di fe ren tesáreas delcono cimien to científico. Enjuniode1998dabacomienzolasegunda fase del proyec to cen tra da en elde sa rrollode colecciones na cionales dere vis tas digitales.Lacolecciónpionerafue SäloBrasil aunqueactualmente secuentaade máscon las colec cio nes de Chile, Cos ta Rica, Cuba, y Es paña. Este mo de lo de de sa rrollo ba sado en infraestructuras nacionales garantiza la continuidad del pro yec to y el crecimien to de la colec ción. Deesta forma, en di ciem bre del año 2000la biblio te ca vir tual con ta ba con unos se sen ta títulos de re vis tas cien tíficas, y ha cia finales de 2002 lacoleccióncontabayacondoscientostítulos.

El sitio web seorga niza en tres mó du los: co lec ción física de re vis tas, ba ses de datos y módu lo es ta dís tico. Una de las ma yo res bon da des del sis te mare si de en quelos tres ele men tos estánin te grados, detal for ma quela bús que dase pue de efectuara partirde diver sas pala bras claves, y se cuen ta con la po sibilidad de consul tarlas es tadísticas quein dicanquére curso web hasidoel másutilizado, cuán tas ve ces sehaacce dido a él o quién ha citado este trabajo científico. Ade más, y mer ced a un acuer do con el ISI, Scie lo tra baja con su mo de lo es ta dís ti co para me dir eluso y el im pac to dela pro duccióncientífica.

Enresumen, lame to dología deScielo se sus ten ta sobre tres pila res de trabajo. El prime rode ellos permitela publica ción elec trónica de ediciones com ple tas delasrevis tas cien tíficas, la orga niza ción de bases de da tos bibliográ ficas y de tex toscompletos, larecupera ción de con te nidos, la preservación de archivos electrónicos, y lap roducción de indicadores estadísticos de uso e impacto de la literatura científica. De he cho los tex tos delos ar tícu los sonen rique cidosdiná mica men te conhipe renla ces hacia bases de datos nacionales o internacionales, según los casos, como por ejemplo,LilacsyMedline.

Elsegundopilar detrabajo en la me to dologíaScielo tienequeverconlaaplicación de dicho méto do a si tios web de colec ciones de re vis tas elec tró nicas. Scielo fa vo rece asílaope ra ción de si tios nacio na les y también de si tios te máticos. Yel terceryúl timo pilarde tra bajo dela me to do lo gía es el de sa rro llo de alianzas en tre los ac to res nacionaleseinternacionales delacomunicacióncientífica, conelobjetivodedifundir, perfeccionarymantenerelmodelo.

En lo que ata ñe a Scie lo Es pa ña (Figura2), pue de afir mar se que se tra ta de una bibliote ca virtual formada poruna pequeña colección derevistascientíficases pañolas, especializadas en Ciencias delaSalud, que estácon formadaporlossiguien testítulos (consultaefectuadaenmayode2003): 
Figura 2

Portal deaccesoaScieloEspañaA nales de Medicina Interna - 26 números

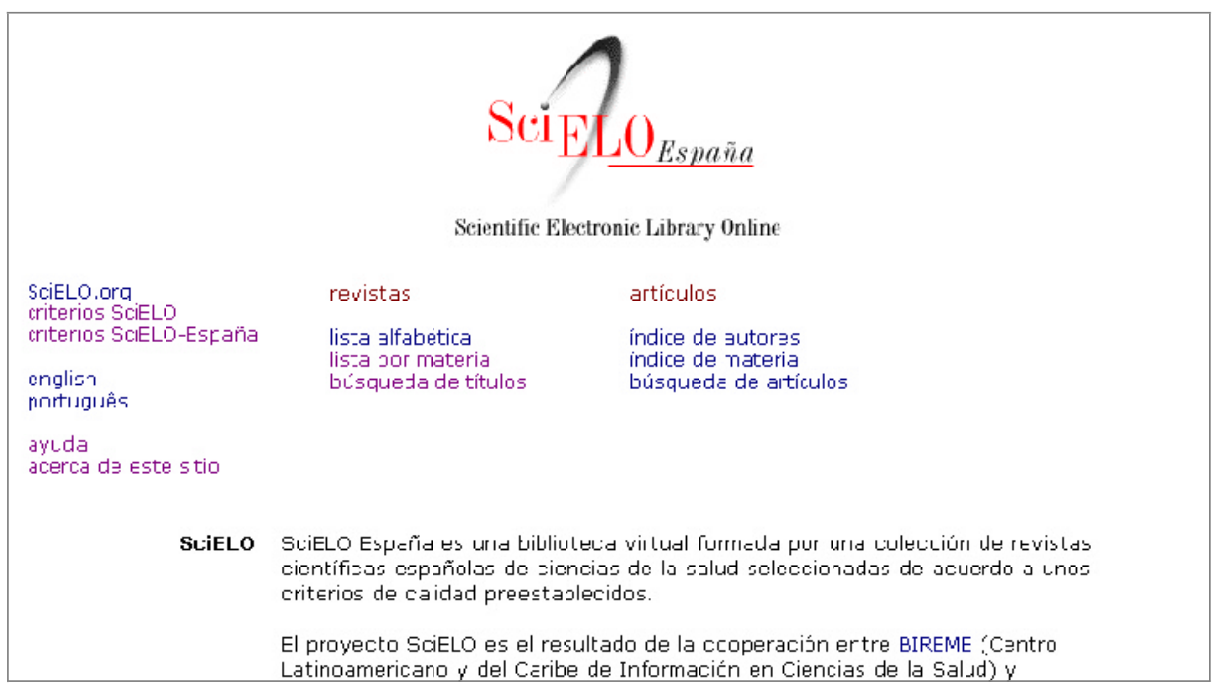

* Ardivos dela Sociedad Españda deOftalmoloǵa - 5 números.

* EducaciónMédica-1 número.

* Gacta Sanitaria- 7 números.

* Medifam- revista de medicina familiar y comunitaria - 22 números.

* RCOE - 8 números.

* RevistaEspañda deSaludPública- 36 números.

* Reista deDiagósticoBidógico- 8 números.

* TheEuropeanjaumal of psydiatry(edición en español) - 4 números.

Los ob je tivos prio ri tarios dela colec ción de Cien cias delaSalud deScielo Es paña sesumanalosobjetivosdelproyectoglobal,ypuedenconcretarseenlos siguientes:

* Contribuir al desarrollo de la investigación.

* Aumentar la difusión de la producción científica nacional.

* Mejorar y ampliar los medios de publicación.

* Evaluar los resultados.

La colección permite el acceso a toda la colección de revistas, a números individuales, yaltextocompletodelosartículos. Comodesarrollos futurosseapuntan:

* Enlaces desde las referencias bibliográficas de los artículos a los registros de las bases de datos especializadas (MEDLINE, Lilacs).

* Conexiones internas a SCIELO.

* La puesta en marcha de un módulo complementario que facilitará informes sobre las consultas realizadas a la colección. 


\section{InvestigacónBiblicteedógia v. 18 No. 37 julio/ diciembre de 2004}

Porsupartelaconsul tauorganizacióndelainformaciónesgratuitaypermite:

* Acceso a la colección de revistas mediante índices alfabéticos de títulos y materias.

* Formulario de búsqueda por palabras del título, nombres de en tida des, luga res de publicación y materia.

* Búsquedas en la base de da tos porau to res, títulos, ma te ria, pa la bras del resur men, etcétera.

La selección de las fuentes sigue unos exhaustivos criterios entre los que se encuen trala evalua ción dela calidad del con te ni do, del co mi téedi to rial,la in clusión de trabajos originales, la pe rio di cidad de publica ción, et céte ra. De esta forma, elcrecimien to dela colec cióncuen ta concriterios de calidadbasados en elProtocolodeeva lua cióndela Base de Da tos IBECS(Ín diceBibliográ ficoEspañoldeCiencias delaSa lud), que fija suactuaciónen tornoalos siguientesítems:

* Revistas que publican predominantemente trabajos originales y de investigación de carácter científico.

* Noseevalúanlastraducciones, niboletinesepidemiológicos,opublicaciones con contenidos institucionales, informativos y comerciales.

Del mis mo modo, la presen ta ción de los tra bajos de in ves tiga ción cuenta con una evaluación de calidad sustentada en criterios de presentación que siguen la Norma UNE50-101-90:

* Adecuación del título a la materia de la revista.

* Mantenimiento del formato en todos los números.

* Indicación de la periodicidad.

* Regularidad de aparición.

* Datos completos en cubierta, portada y sumario.

* Datos identificativos de la revista y del artículo en cada página del texto.

* Otras informaciones: trabajo de los autores, etcétera.

Elcontenidocientíficodelostrabajosseapoyaenlos siguientescriteriosde calidad:

* Composición del comité editorial o consejo de redacción.

* Normas de publicación.

* Calidad científica y variedad de contenidos.

* Apertura a autores de todo el país y de distintas instituciones.

- Actualidad de las referencias bibliográficas incluidas en los artículos.

Ladifusióndelos trabajos siguelossiguientescriteriosdecalidad:

* Publicación habitual de artículos de autores extranjeros.

* Resumen en español e inglés.

* Palabras clave en español e inglés.

* Difusión de la publicación en Internet.

* Indización en bases de datos y repertorios bibliográficos (nacionales e internacionales). 
* Indización en el Jaumal Citation Repats(ISI).

* Indiza ción en INSIDE (Centro de Su minis tro deDo cumentos dela Biblio teca Británica).

Laes pecificidad dela fuen te dein forma ción digital es muyalta. Dehechoelproyecto busca, acortoplazo, aumen tarla visibilidad,laaccesibilidadyla credibilidadnacional e internacional de las publicaciones científicas de América Latina y el Caribe, para lo cual sigue una metodología de publicación, integrada en Internet, sobre colecciones nacionales yregionales derevis tas científicas. Deahíquepuedaafirmarseque suobjetivo, alargoplazo, es contribuiral aumento delimpactodelaproducción científicade estas regiones. Portan toestamosauditandounafuen tedein forma ciónintrínsecamente valiosa,pues setratadeunserviciodeI+Dque:

* Promueve el intercambio y mejora de la comunicación científica.

* Identifica, es timulayde sarrollaunacolección-núcleoderevis tascientíficasy las hace más accesibles.

* Cuida que las revistas tengan un estándar de calidad de primera línea.

Di cho es tán dar de ca li dad de las re vis tas tie ne su ori gen en los 33 cri te rios for males, internacionalmente establecidos, que toda re vis ta debe cum plir si su pre ten sión es sobrepasar el ámbito local y entrar a formar parte del universo digital de Scielo. Cadaunodees tos re quisi tos fuees tablecidoporLa tindex, Sis temaregionaldein formación en línea para re vis tas cien tíficas de América La tina, ElCaribe, Es pañay Portugal, y está claramente estipulado en la sede web de Scielo. Si la revista postulante cum pleal me nos con 25 de los re que ri mien tos pasa a la eta pa de eva lua ción de con tenido, dondeunComité, es table cidoporCONACYT se gún el tema de la re vis ta, se encargadeevaluarlacalidaddelos artículos.

Respecto al alcance de la colección, se incluyen revistas científicas que publican principalmente artículos que son resultadodeinvestigaciones científicasoriginales. Sesigue el pro ce so de revisión por pa res. La colec ción de cada títuloin dividualdebe ini ciar se a partir delaño 1997, o del primernú me ro, para re vis tas crea das des pués de 1997, y las revistas de Scielo no tienen que ser publicadas necesariamente en papel. Un caso es pe cial es EJB(EløtranigamalofBiotedndogy) dela Univer sidad Ca tólica de Valpa raíso. Se tra ta dela úni ca pu bli ca ción de Scie lo que sólo exis teen for ma to electró nico. Otro caso cu rio so es eldela re vis ta EURE (RevistaLatinomericanadeEstudios Urbanos Reejonales) de la Pon ti fi cia Uni ver si dad Ca tó li ca de Chi le, que na ció en la déca da de los 70 y en 1998 fue in vi ta da a par ti ci par en Scie lo, y tras dos años de par ti cipa ción, su edi tor, Gon za lo Cá ce res, afir ma ba que se ha bía lo gra do el sue ño de cual quier revista: ser indizada en ISI, el mayor catálogo en línea de revistas científicas. Además EURE sehabía convertidoenlaúnicarevis tahis panoparlantedelámbitourbanís tico regionalin diza da enelISI, con locual se cons ti tu yó en un re fe ren te cla ro en su ámbito, para la publicación de trabajos científicos chilenos y latinoamericanos, pues publicar en una revista ISI le ofrece más posibilidades al científico para ganar proyectosyobtenerpremiosdeproductividad. 


\section{InvestigacónBiblicteedógica v. 18 No. 37 julio/ diciembre de 2004}

Segúnlos da tos ofrecidos enlaIReunión RegionaldeScielo7 la colec ción de revistasdeestaplata formapresentaelsiguienteperfil:

Tabla 1

Distribución editorial de la colección de Scielo, por áreas de conocimiento

\begin{tabular}{|l|c|c|c|c|c|c|c|c|c|}
\hline Área científica & Total & $\begin{array}{l}\text { Sociedades } \\
\text { científicas }\end{array}$ & $\%$ & Univ. & \% & $\begin{array}{c}\text { Inst. de } \\
\text { pesq. }\end{array}$ & \% & 0tros & \% \\
\hline Agranias & 14 & 6 & 42,8 & 4 & 28,5 & 2 & 14,8 & 2 & 14,8 \\
\hline Biológicas & 21 & 12 & 57,1 & 3 & 14,2 & 2 & 9,5 & 4 & 19 \\
\hline Salud & 32 & 21 & 65,6 & 6 & 18,7 & 3 & 9,3 & 2 & 6,2 \\
\hline $\begin{array}{l}\text { Exac tas y de la } \\
\text { Tiera }\end{array}$ & 14 & 12 & 85,7 & 1 & 7,1 & 1 & 7,1 & - & - \\
\hline Humanas & 17 & 2 & - & 12 & - & 1 & - & 2 & - \\
\hline $\begin{array}{l}\text { Sociales y } \\
\text { aplicadas }\end{array}$ & 3 & - & - & 1 & 33,3 & 1 & 33,3 & 1 & 33,3 \\
\hline Ingenienías & 7 & 6 & 85,7 & 1 & 14,3 & - & - & - & - \\
\hline $\begin{array}{l}\text { Lingüística, } \\
\text { letras y arte }\end{array}$ & 1 & 1 & 100 & - & - & - & - & - & - \\
\hline
\end{tabular}

Fuente: Elaboración pro pia a par tir de los da tos fa ci li ta dos en la I Re u nión Re gio nal.

Los da tos de la Tabla1 permiten de finir tresgrupos edito riales. Enelprimeropredominan como editores las sociedades científicas, y engloban las áreas de ciencias agra rias, cien cias bio ló gicas, cien cias dela sa lud, cien cias exac tas y dela tie rra, eingenierías. Enelsegundogrupopredominan comoeditoraslasins titu cionesuniversita rias y abarcan las áreas de cien cias hu ma nas y lin gǘs tica, le tras y arte. El tercergru po consiste en un grupoindefinido, que in clu ye elárea de cien cias so cia les y apli ca das, pero que no se de can ta pornin gún gru po edi to rial, al con tarcon un ítem edi ta do por lassocieda des científicas, yotroporlasuniversidades. Estosda tosnos sugie renestas tresgrandescaracterís ticasdelacoleccióndeScielo:

* Ninguna revista de Scielo es editada por un editor comercial.

* En el cam po de las cien cias exac tas y de la vida, y sus tec no lo gías, se de fi ne un claro predominio de las revistas editadas por sociedades científicas.

* Enel cam po de las cien cias hu ma nas y so cia les pre do minan las re vis tas publicadas por las instituciones universitarias.

7 IReuniónRegional delaRedSäloInternationalConference on Scientific Elec tro nic Publishingin DevelopingCountries. [Fe cha de ac ce so 20 di ciem bre 2002]. Dis po ni ble en el do cu men to web: http://www.icsep.info/. 


\section{Evaluación de Scielo}

Laevaluacióndel recurso selec cionado,Scielo, se susten ta en primerlugarteniendo en cuen ta el análisis an te rior men te ex pues to y quein cide en el de sa rrollo que hemos llevado a cabo del protocolo ISI, puesto ya en escena en varios trabajos y con muy buenos resultados. ${ }^{8}$ Dichopro to coloseorganizaen pa rá me tros:laspropieda des o características de la fuente de información a evaluar, los indicadores y los elementos dela pro pia fuen te que se van a con side rarparacada una de las ca rac te rís ticas anteriores.Elprotocoloquedaríaorganizadocomosigue:

\section{Parámetro 1. Autoría y solvencia}

Indicadoresrelacionados conlaadecuacióny solvenciadela fuente:responsabili dad y au to ría de la in for ma ción; solven cia del au tor en el tema de la fuen te; da tos de identificaciónyposibilidaddecomunicaciónocontactoconelmismo.

\section{Pará metro 2. Contenido}

Indicadores relacionados con el valor intrínseco de la in formaciónque ofrecela fuen te y su vo lu men: va lorin trín se co dela in for ma ción y pres ta ción de servicios de valoraña dido; volu men de in for ma ción que ofre cela fuen te en re la ción conla es pecificidad del re cursoyel temaquetrata;calidadyexhaustividaddelainformación con baseenuncon troly un tra ta mien to documental previo; y frecuen cia de actualización delainformación.

\section{Parámetro 3. Acceso a la información}

In dica do res rela ciona dos conlasopcio nes denave ga cióny re cupe ración delainformación que le ofrece la fuente al usuario: navegación estructurada y de calidad; orien ta ciones decon tex to en la nave ga ción; je rarquización previa deloscon teni dos según su importancia relativa; análisis de las opciones de búsqueda; inclusión de

8 Sirvan de mues tra los siguien tes tra ba jos:

- Ayu so García,Ma Dolores, PeñalverMartínez,Ángel,y MartínezNavarro, Vic toria:"Evaluación de fuen tes de in forma ción para servicios de re fe ren cia en In ternet. Una propues ta a par tir de "Pregun te, las biblio te cas res pon den'”'. En, ICongesoNacionaldeBibliotecasPúblicas Labibliotecapública, portal delasociedaddelainformacón Va len cia, 29-31 oc tu bre 2002. [Fe cha de ac ce so 8 fe bre ro 2003]. Dis po nible en eldo cu men to web:

http://www.mcu.es/lab/bibliote/travesia_actas/pdf/sumario.pdf.

- Ayu so García, $\mathrm{M}^{\mathrm{a}}$ Do lo res: Red te má ti ca de do cen cia so bre: crea ción de ser vi cios y sis temas de in forma ción digital (S²ID) paralaSo ciedad del Cono cimien to. Universidad CarlosIIIde Ma drid, UniversidaddeSalamanca, UniversidaddeMurcia,PontificiaUniversidadCatólicade Perú, Pon tificiaUniversidadJa ve ria na deBogo tá,y Universidad deLaHabana.PonentedelMódulo: “Au ditoría de recursos informativos y sistemas de información digital. Publicaciones digitales y normalizaciónderecursos". Diplomadode "Sis temasyservicios dein formacióndigital".Bogotá, 9-13 de junio de 2003.

- Ayu so García, Mª Doloresy MartínezNavarro, Vic to ria: "La edición digitaldelBole tín Oficialde la Región de Murcia: pa rá me tros e in dica do res para su evalua ción", en AnalesdeDoamentación vol. 6, 2003. 
tablas decontenidos, sumariosomapasdenavegación;índicescronológicos,temáticos, geográficos u onomásticos;navegación semán tica;y con sis tencia en el sis te ma deseñalesdenavegación.

\section{Parámetro 4. Ergonomía.}

Indicadores rela ciona dos conlacomodidady facilidaddeutilización delafuente dein forma ción: relación figu ra / fon doen la web; tipografía eimágenes emplea das; márgenesyespaciadosentrelospárrafos.

\section{Parámetro 5. Luminosidad}

Indicadores relacionados con la presencia y calidad de enlaces externos: enlaces con otras webs; evalua ción de di chos en la ces y ac tualiza ción; presen ta ción dein formacióndevalorañadidosobredichosenlacesexternos.

\section{Parámetro 6. Visibilidad}

In dica dores rela ciona dos con el nú me rodeenlaces que re cibela fuente dein formación deotrosrecursosex ternos:inclu sión enelelemento HEAD de meta eti que tas como "Author", "Keywords" y "Description"; descripción adecuada de la fuente median teun sis temaavanzadodeme ta da tosen el elemen toHEAD; establecimiento de un título identificativo así como de párrafos iniciales explícitos con respecto al contenidodelrecurso;identificacióndelafuen temediantela URL, y nú me rodepági naswebderecursossimila resocomparablesqueapuntanalrecursoevaluado.

En cuan to al sis te ma de pun tua ción, para este caso con cre to las au to ras han convenido en asignarde 0 a 3 pun tos a cada pa ráme tro se gún la ade cua ción de susin dica doresala situa ciónóptima (estándar), excepto aquellosindica doresque,porsunaturaleza -cum plir o no cum plir el es tán dar-se re co mien da pun tuar o bien 0 ó bien 1 . Paralosindica dores de 0 a 3 las au to ras han llevadoa cabolasiguien tein ter pretación ypropuestomejorasenaquellaspuntuacionescomprendidasentre0y2.

\begin{tabular}{|c|l|}
\hline Puntuación & \multicolumn{1}{|c|}{ Interpretación } \\
\hline 0 & Error severo \\
\hline 1 & Error \\
\hline 2 & Estándar correcto,aunquemejorable \\
\hline 3 & Excelente(Estándar óptimo) \\
\hline
\end{tabular}

Scielo. Colección de España. [Fe cha de ac ce so 19 mayo 2003]. Dis po ni ble en el do cu men to web http://Scielo.isciii.es:90/Scielo.php.

* Fecha de inicio: 19 de mayo de 2003.

* Fecha de finalización: 23 de mayo de 2003.

* Metodología empleada: protocolo de evaluación del ISI desarrollado por las autoras. 
Figura 3

De tal le de los cré di tos del re cur so. E- mail de con tac to con Scie lo Es pa ña

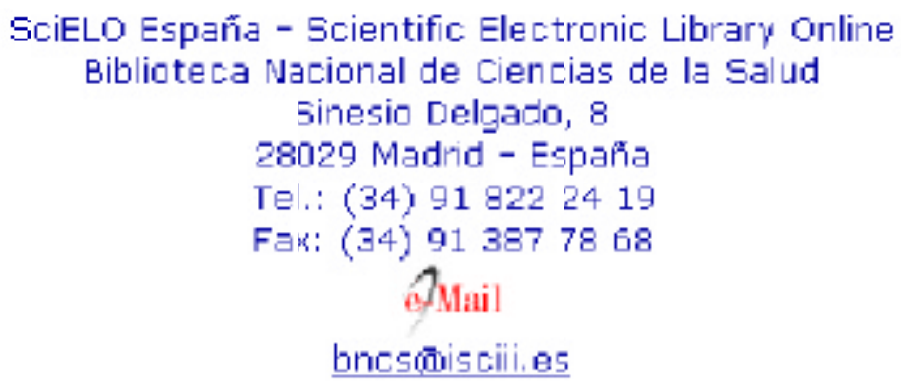

\section{Pará metro Autoría}

* Indicadores relacionados con la adecuación y solvencia de la fuente: responsa bili dad y au to ría de la in for ma ción; solven cia del au tor en el tema dela fuente; datos de identificación y posibilidad de comunicación o contacto con el mismo (Figura3).

* Puntuación prescrita: 0-3.

* Puntuación para Scielo: 2.

Observaciones:

> Lafuen tedigitalevaluada presenta unnivelacep tabledede sarrollodelos indicadores de autoría / fuente. Esto es habitual en web institucionales con proyectos de cooperación. No obstante, podría ofre cerse un formur lario para el envío de consultas o sugerencias.

\section{Pará metro Contenido}

* Indicadoresrelaciona dos conelvalorin trínse codelain formaciónqueofrece la fuen te y su vo lu men: va lor in trín se co de la in for ma ción y pres ta ción de servicios de valoraña di do; volu men de in forma ción que ofre cela fuen teen relación con la especificidad del recurso y el tema que trata; calidad y exhaustividad de la información con base en un control y un tratamiento documental previo; y frecuencia de actualización de la información (Figra 4).

* Puntuación prescrita: 0-3.

* Puntuación para Scielo: 3.

* Observaciones:

> Lafuentedigitalevaluadapresentaperfectamentedesarrolla doslosin di cadores del parámetro contenido. La especificidad del recurso es muy alta, así como la calidad, que sigue pautas internacionales de medición. 


\section{Figura 4 \\ Detalle de la coleccióndeScieloEspaña}

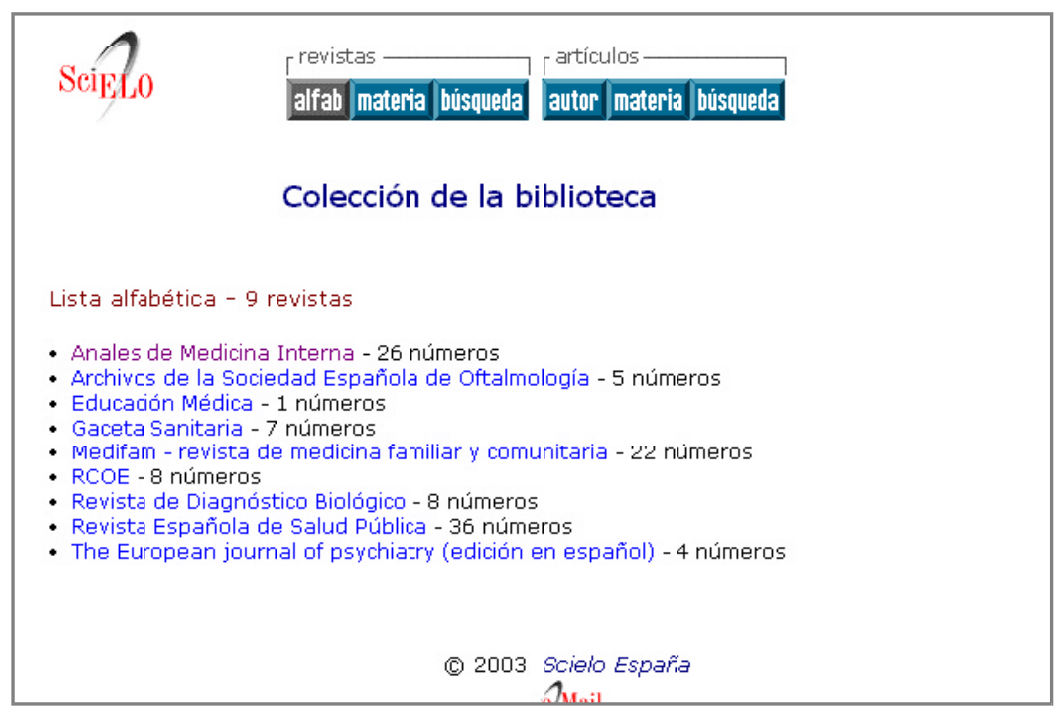

\section{Parámetro Acceso a la información}

* Indicadores relacionados con las opciones de navegación y recuperación de la in forma ción quele ofre cela fuen te alusua rio: na ve ga ción es truc tu ra day decalidad; orientaciones de contexto en la navegación; jerarquización previa de los contenidos según suimportancia relativa;análisis delasopciones debúsqueda; inclusión de tablas de contenidos, sumarios o mapas de navegación; índices cronológicos, temáticos, geográficos u onomásticos; navegación semántica, y consistencia en el sistema de señales de navegación (Figura5).

* Puntuación prescrita: 0-3.

* Puntuación para Scielo: 3.

* Observaciones:

$>$ Como en los parámetrosanteriores la evaluación es muy positiva. El accesoa lain forma ción se ha de sa rrolla dopen san do en elusua rio es peciali zado de la fuente de información, lo que contribuye a adecuar las expectativas creadas para los usuarios.

Otras observaciones:

$>$ Ofrece índices de consulta de autores y materias.

$>$ Ofrece formulario de búsqueda.

$>$ Sehadesarrolladolana vegaciónsemánticaenlos regis tros bibliográficos.

$>$ Las descripciones bibliográficas se acogen a la normativa internacional.

$>$ Seofrecenlos tex tos com ple tos delos artículos en va rios for ma tos: html o pdf. 
Figura 5

Detalle del formulario de consulta,ScieloEspaña

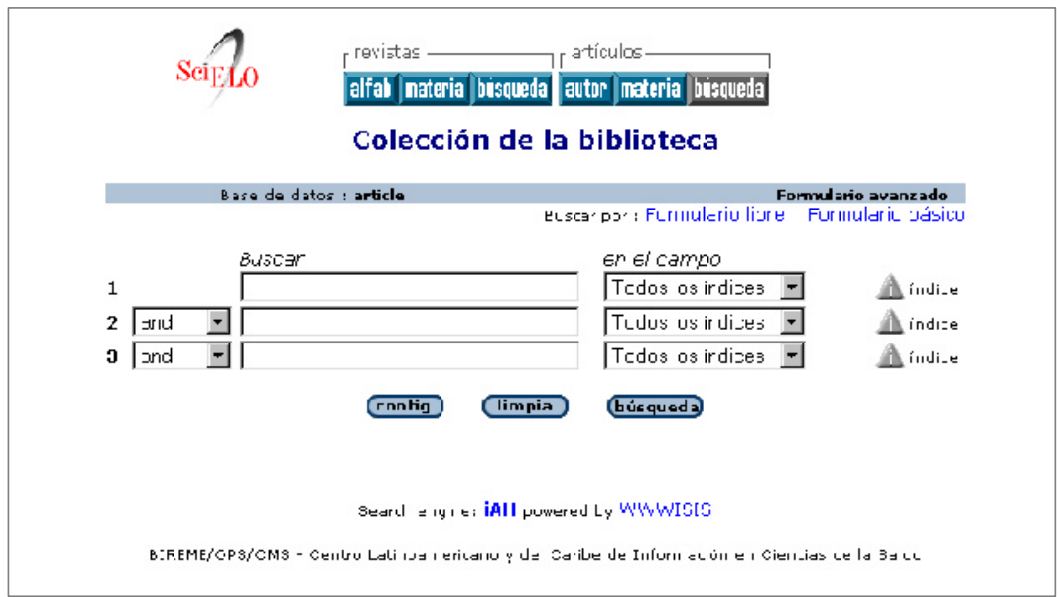

Se facilita información para realizar correctamente, según la norma ISO 690, la referencia bibliográfica.

\section{Parámetro Ergonomía}

* Indicadores relacionados con la comodidad y facilidad de utilización de la fuente de información: relación figura / fondo en la web; tipografía e imágenes empleadas; márgenes y espaciados entre los párrafos / agradable y fácil (Figura 6).

\section{Figura 6}

\section{Detalle de con sul ta del resu men de un ar tículo}

\section{Scip \\ ¡ Đrtículos —búsquedade artículos - \\ sumario anterior próximo autor materia búsqueda home alfab}

\section{Archivos de la Sociedad Española de Oftalmología \\ ISSN 0365-6691 versión impresa}

Resuriteri

VILLARRUBIA CUADPADO, A, GALLARDO GALERA, JM, BERGILLOS ARILLO, M et al. Lente ZSAL-4 para la corrección de la alta miopía. Arch Soc Esp Oitalmol, dic. 2002, vol.77, no.12, p.661-667. ISSN 0365-6691.

Objetivo: Estudiar la eficacia, segıridad y predictibilidad del implan:e de lente ZSAL-4 para la corrección quirúrgica de la alta miopía. Método: Estudiamos retrospeztivamente 28 ojos de 19 pacientes er los que se implantó una lente ZSAL-4. Los datos recogidos 


\section{Figura 7}

De tal le de la pá gi na "En la ces de in te rés", sec ción "Ini cia ti vas de pu bli ca ción electrónicadeperiódicos"

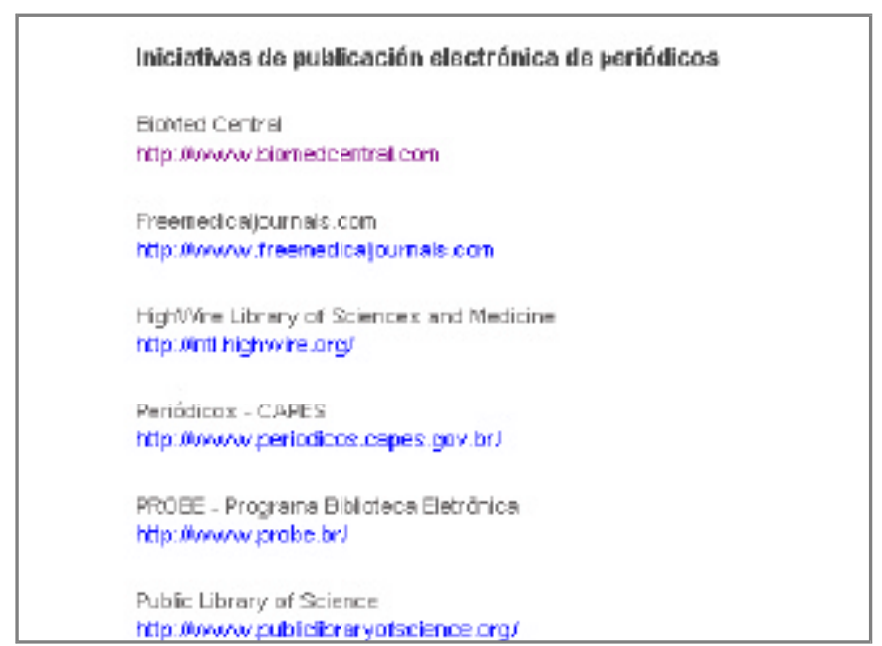

* Puntuación prescrita: 0-3.

* Puntuación para Scielo: 3.

* Observaciones:

$>$ Buena rela ción figu ra / fon do en las pá ginas web; bue na ti po gra fía, már genes y espaciados; y escaso uso de imágenes.

\section{Parámetro Luminosidad}

* Indicadores relaciona dos con la pre sen cia ycalidad de enlaces ex ternos: en la ces con otras webs; evalua ción de di chos en la ces yactualiza ción; presen tación de información de valor añadido sobre dichos enlaces externos (Figura 7).

* Puntuación prescrita: 0-3.

* Puntuación para Scielo: 1.

* Observaciones:

$>$ Dotardeluminosidadala sededeScielo de sa rrollan dolas sec ciones deenla ces de in te rés más allá de un lis ta do deURLs, y buscarinformación complementariasobrelos recursos queseofrecencomocandidatos devisita.

\section{Parámetro Visibilidad}

* Indica dores relacio na dos con el nú me rode en la cesque re cibela fuen te deinformación de otros recursos externos, y trabajo con metadatos.

* Inclusión en el elemento HEAD de metaetiquetas como "Author", "Keywords" y "Description"; descripción adecuada de la fuente mediante un sistemaavan za do de me ta da tos en el ele men toHEAD; es tablecimien todeuntítulo 
identificativo así como de párrafos iniciales explícitos con respecto al contenidodel recurso;iden ti fica ción dela fuen temedian tela URL; y nú me ro de páginas webs de recursos similares o comparables que apuntan al recurso evaluado.

Puntuación prescrita: 0-3.

Puntuación para Scielo: 1.

Observaciones:

$>$ Se precisa un mayor desarrollo del elemento HEAD, ya sea para contar con un sistema de metaetiquetas como "Author", "Keywords", y "Description"; o bien con un sistema avanzado de metadatos, como Dublin Core, muy extendido en el ámbito académico-científico.

Para conocer si este recurso web está enlazado desde otras páginas, empleamos los motores de búsqueda Google, Altavista, Hotbot, Infoseek, y Lycos. Y, concretamente, la opción que nos permite saber cuántas páginas web apun tan a laURL de Scielo, para lue go cal cu lar la me dia de en la ces con este recurso, tal y como lo muestra la gráfica siguiente (Gráfico1).

* La media de vi sibilidad deScielo se cru za con las ob tenidas en se des web dela misma categoría o naturaleza: consorcios de bibliotecas sanitarias con servicios de revistas digitales.

> Consorcio de bibliotecas de ciencias de la salud de Pennsylvania: www.hslc.org

$>$ Red de información sanitaria del Estado de Arizona: www.azhin.org

$>$ Red de in for ma ción sa ni ta ria del área de Ro ches ter, en Nue va York (Miraclenet): www.urmc.rochester.edu/miraclenet.

Gráfico 1

Popularidad de la sede web de Scielo

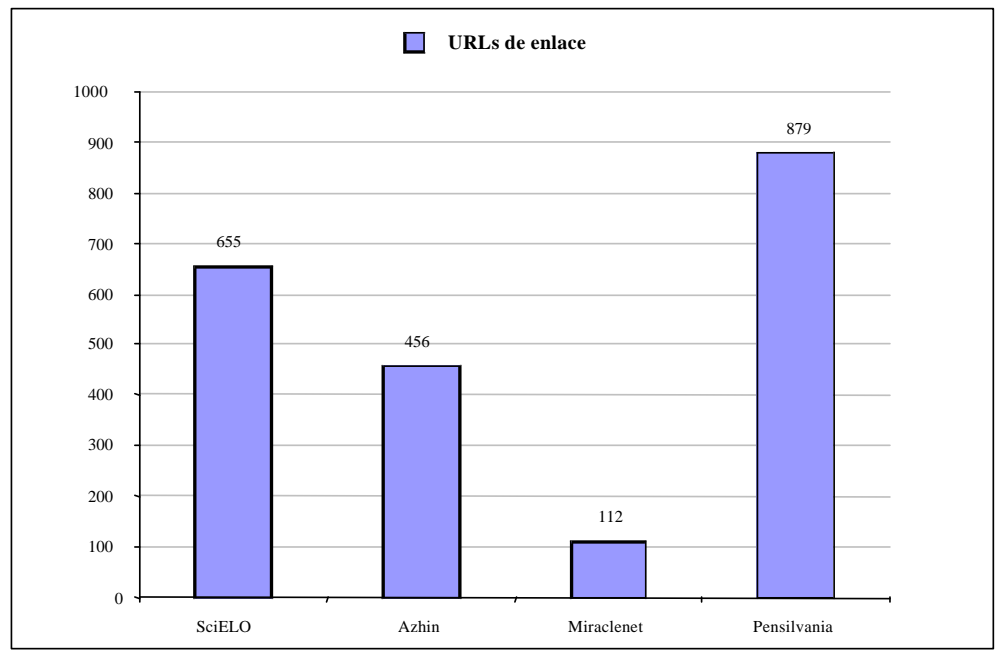

Fuente: Elaboración propia. 
Del resultadoobtenidopue deconcluirsequela sedewebdeScielocuentacon un buen ín di ce de en la ce des de otras pá gi nas web, en con so nan cia con los resultados obtenidos para el resto de sedes puntuadas.

\section{CONCLUSIONESY PROPUESTA DE MEJORA}

Ellími te de ex ten sión so bre el con teni do de este tipo de traba jo nos im pi de de sarrollarunin forme ex haus tivocomo resulta do final, demodoque se aportanlos da tos de forma suscinta. La evaluación del Proyecto Scielo ha puesto de manifiesto los puntos fuertes y débiles de esteserviciodeinformacióncientíficapublicadoenInternet, como ya hemos indicado en el epígrafe anterior. Por tanto, ha corroborado la afir ma ción con la que las au to ras co men za ban el tra ba jo, la au di to ría de fuen tes y recur sos dein for ma ción publi ca dos en la Red es uno de los es ce na rios de tra bajo de la evaluación.

Teniendo en cuenta la afirmación anterior, y finalizada la sesión de evaluación, tras la cual los evaluadores, han elaborado una lista de puntos débiles y fuertes, comien za la ex plica ción de és tos con formea los principiosoes tán da res decalidad que describeelprotocoloparacadain dicador.Elanálisisdecadapun to débil,oindicador suscep tible demejo ra, seha derealizarporseparadoyno en con jun to. Setra tade evitarque se re pitan los erro res en el re diseño dela web y permi tir la solu ción de problemas concretos sin necesidad de rediseñar todo el sitio porcomple to. Laje rarquiza ción dela grave dad delos pro ble mas, opun tos débiles, sin duda facilita, laaplica ción posteriordemedidascorrectoras. Dichagravedadesmedidaportresfactores:

1.- La frecuencia con la que el problema ocurre.

2.- El impacto del problema cuando sucede.

3.- La persistencia del problema, esto es, si el problema se resuelve la primera vez que se use el sitio web o aparece repetidamente.

Toda vez que en la evaluación de Scielo han sido aplicados los indicadores propuestos, apareceunpeso significativodelos denominadospuntosfuertesfrentealos débiles, es pe cialmenteenloquese re fiereala me to dología de trabajoconlaspublicacionescientíficas. Portantopodría con cluirse afirman doque elproyec to Fapesp/ Bi reme con tem pla el de sa rrollo de una me to do lo gía co mún para la pre pa ra ción, el al macenamiento, la extracción y la evaluación de publicaciones electrónicas que empleanlastecnologíasdelainformación.

A par tirdeestame todología de trabajocooperativolapublicaciónelectrónica, ha conseguido promover una renovación en el proceso de la comunicación científica tradicionalalin tegrareluso tra dicionaldelapublica ción con elcon trolbibliográfico, lapreservaciónyelmantenimien todelascolecciones, yelfactordeimpactodeéstas.

Según todo lo an te rior, el proyec toScielo podría de finirse comola tran sición dela literaturacientíficadeAméricaLatinayElCaribedesdelagalaxiaGutemberghas tala sociedad de la información y el conocimiento. Todo este trabajo se sustenta sobre 
una pla ta for ma di gitalquele ofre ce cada vez un ma yorcam po de di fu sión ala co munidad cien tífica merced a los acuer dos que sehan sus critocongrandes bases de da tos como ISIyMedline.

Como no po día serde otra forma, los ojos dela co mu nidad cien tí ficain ter nacionalcomienzanamirarhacialacienciala tino americana. Elcientíficola tino americano sabeque sipublicaunartículo en elárea médica, en Scieloau to máticamenteseen vía una co pia de éste a Medline. Medli nele in cor po ra a su sis te ma y permi tela con sul ta a tex toíntegrodedichotrabajo. Ademássial gunadelascitasquefigu ranenScielopertenece a una re vis ta que está en Medli ne, el sis te ma la vin cu la para que el usua rio conozca el tex to completo, otrodelos in dicadoresde calidadobservados.Los acuerdos con ISI vanen la mis malínea de tra bajo, de tal forma que siun in ves tiga dor sein te re sa por los artículospublicados en algu na delas revis tasin te gran tes delProyecto, tenga laopcióndeen traraScieloyconsultareltrabajoín tegro.

La auditoría de Scielo también ha puesto de relieve algunos puntos débiles de este recursoin formativo, especialmente elquetienequeverconlaoperacionalidad detrabajodel proyec to. Como mos tra ronal gunos delos parámetros evaluados, podríanllevarse a cabo de sa rrollos enla publica ción digital que mejo ra rían su ca li dad. A esterespec to se ha he cho re fe ren cia en la au to ría a la exis ten cia de un for mula rio de en vío de suge ren cias, yen cuan to a la lu mino sidadala existen cia de un directo rio oca tálogo que analiceycomentelosenlaces recomendados;porúl timo, enelparámetrovisibilidad se hadetectadolanecesidad de de sarrollarlas metaetique tas delelemen to HEAD, o bien ladeutilizarunsistemaavanzadodemetadatoscomoDublinCore.

\section{FUEN TESYRE CURSOS}

Abbot, C. (1994): Pefomancemesurement in library and information services Londres: Aslib.

Ayuso García, Mª Dolores (2001). "Bibliografía, Información y Conocimiento. Del método bibliográfico a la normalización y evaluación de recursoselectrónicos", en HomenajeaJuanA. Sagadb EstudiosdeBiblio grafía yFuentes deInformaión Madrid: Universidad Complutense.

Ayuso García, Mª Do lo res y Martínez Na va rro, Vic to ria (2003a): "El documento elec trónico ylali te ra tu ra gris:la co mu nica cióny di fu sión del conocimientocientíficoen In ter net", en AdasdlasJarnadasAndaluzas deDoumentadón, JADOC'03.

--. (2003b): "La con so li da ción de las nue vas -ac tua les for mas de co municación cien tífica enlaSo cie dad delConocimien to", enActasddICon geso Intemacional sobre Teendoǵa Docmental yConoimienta

Beaudiquez, Marcelle (1998): GuideGenealedebibliographie Methoddogeet pradique $2^{\mathrm{a}}$ ed. París: Saur, p. 34-38. 
Bi blarz, D. ; Bosch, S. y Sug net, C. (ed.) (2001): Gui detoli braryuserneedsas sessment for integrated information resaurce managment and colletion deve lopnet. Lanham: Scarecrow Press.

Burk, C.F. y Hor ton, F. W. (1988):Infomapacompletegiidetodiscoveringromp rateinfomation resares. Englewood Cliffs: Prentice Hall.

Coo ke, A. (2001) $2^{a}$ ed.A giidetofindingqualityinformationantheInternet:sdee tionanevaluationstrateges Li brary Asso cia tion Pu blishing. No so tros hemos utilizado la edición de 1999.

Henczel, Sue (2000): Theinformation audt: a pradical gaide Munic: K.G. Saur.

- -. (2001): "The information audit as a first step towards effective knowledge management: an opportunity for the special librarian", en Inspd, 34, no 3-4, p. 210-226.

Meneghini,Rogério(1998): “Avallaçãoda produçãocien tíficaeo proje to Scielo", en Ciêna da infomação V. 27, n 2. [Fecha de acceso 3 abril 2000]. Disponible en el documento web: http://www.Scielo.br.

Orna, Eli za beth (1990):Pradicalinformationpolides hontomanageinformation flowin arganisations Londres: Gower.

--. (1994): "The value of information", en Thevalueandimpadofinforma tion Londres: Bowker-Saur, p. 9-77.

- -. (1996): "Valuing information: problems and opportunities", en The fauth resarce infomation anditsmanagement. Londres: Aslib; Gower, p. $18-40$.

- -. (1999): Pradical infomation pdidies Londres: Gower.

Parker, Abel Laerte $\boldsymbol{\notin}$ al. (1998): "Scielo: una metodología para la publicação elec trônica", en Ciênaadainformaşão V.27, $n^{\circ}$ 2. [Fe cha de ac ce so 3 abril2000]. Dis po nible en eldocumen toweb:http://www.Scielo.br.

Parker,AbelLaerte (1998): "Scielo, scien tific elec tro niclibrary on line", en Icsupressworkshop Abril 1998. [Fe cha de ac ce so 4 mayo 2000]. Dis po nible en el documento web:

http://www.bodley.ac.uk/icsu/packerppr.htm.

--. (1999): "An electronic publishing model fordevelo ping, coun tries", en Electronicpublishing1999:redefiningtheinformationdhainnenwaysandvoice Ronneby, Sweden. [Fecha de acce so 10 no viem bre 2001]. Dis poni ble en el documento web: http://www5.hk-r.se/elpub99.nsf. 
- -. (1999): “The Scielo project Latin America and Caribbean: advances and cha llen ges of an emer ging mo del for elec tro nic pu blishing in de veloping coun tries", en Intemadional neworkfortheavai labilityofscientificpt blications [Fecha de acceso 10 noviembre 2001]. Disponible en el documento web:

http://www.inasp.org.uk/psi/scpw/papers/packer.html.

Scielo. Brazil. Scientific electronic Library Online. (Fecha de acceso 20 Febrero de 2004). Disponible en documento web: http://www.scielo.br.

Scielo. Co lec ción de Es pa ña (Fe cha de ac ce so, 19 Mayo de 2003) Dis ponible en el documento web: http://Scielo.isciii.es:90/Scielo.php.

Scielo. Methodblogunderdeudqument. [Fe cha de ac ce so 20 sep tiem bre 2003]. Disponible en el documento web: http://www.Scielo.br/fppe/projeto/emetodo.htm.

Soy, Cristina (2000): Auditona dela infomadó(reurs detrònid). Barcelona: UOC.

Usunáriz Garayona,Jesús María: "Dosiniciativas parala di fusióninterna cional de revistas científicas latinoamericanas". [Fecha de acceso 4 febrero 2001]. Disponible en el documento web: http://www.unav.es/limh/LIMO/limo.htm.

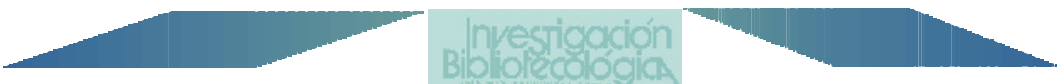

